\title{
Behavioral Analysis of Aggregation in Multicriteria Decision Aid
}

\author{
Jean-Luc Marichal* \\ Revised version
}

\begin{abstract}
The most often used operator to aggregate criteria in decision making problems is the classical weighted arithmetic mean. In many problems however, the criteria considered interact, and a substitute to the weighted arithmetic mean has to be adopted. It was shown that, under rather natural conditions, the discrete Choquet integral is an adequate aggregation operator that extends the weighted arithmetic mean by the taking into consideration of the interaction among criteria. However, since this operator is constructed from coefficients (weights) whose meaning is not always very clear for the decision maker, it is useful to define from these coefficients some indices that offer a better understanding of the behavioral properties of the aggregation. We present and discuss the following indices: the global importance of criteria, the interaction among criteria, the influence of the criteria on the aggregation, the tolerance of the decision maker, and the dispersion of the weights on the criteria.
\end{abstract}

\section{Introduction}

Let us consider a finite set of alternatives $A=\{a, b, c, \ldots\}$ and a finite set of criteria $N=\{1, \ldots, n\}$ in a multicriteria decision making problem. Each alternative $a \in A$ is associated with a profile $x^{a}=\left(x_{1}^{a}, \ldots, x_{n}^{a}\right) \in \mathbb{R}^{n}$, where, for any $i \in N, x_{i}^{a}$ represents the partial score of $a$ related to criterion $i$. We assume that all the partial scores are defined according to the same interval scale, that is, they are defined up to the same positive linear transformation. Particularly, this will enable us to embed the scale in the unit interval $[0,1]$.

From the profile of any alternative $a \in A$, one can compute a global score $M\left(x^{a}\right)$ by means of an aggregation operator $M: \mathbb{R}^{n} \rightarrow \mathbb{R}$ which takes into account the weights of importance of the criteria. Once the global scores are computed, they can be used to rank the alternatives or select an alternative that best satisfies the given criteria.

Until recently, the most often used aggregation operators were the weighted arithmetic means, that is, operators of the form

$$
M_{\omega}(x)=\sum_{i=1}^{n} \omega_{i} x_{i}
$$

*Department of Management, University of Liège, Boulevard du Rectorat 7 - B31, B-4000 Liège, Belgium. Email: jl.marichal[at]ulg.ac.be 
with $\sum_{i} \omega_{i}=1$ and $\omega_{i} \geq 0$ for all $i \in N$. However, since these operators are not able to model in any understandable way an interaction among criteria, they can be used only in the presence of independent criteria. They are not appropriate for the aggregation of interacting criteria.

In order to have a flexible representation of complex interaction phenomena between criteria (e.g. positive or negative synergy between some criteria), it is useful to substitute to the weight vector $\omega$ a non-additive set function on $N$ to define a weight not only on each criterion, but also on each subset of criteria. For this purpose the concept of fuzzy measure [23] has been introduced.

Now, a suitable aggregation operator, which generalizes the weighted arithmetic mean, is the discrete Choquet integral, whose use was proposed by many authors (see e.g. [6] and the references there). Of course, the large flexibility of this aggregation operator is due to the use of a fuzzy measure, which makes it possible to model interaction phenomena existing among criteria. However, the meaning of the values of such a fuzzy measure is not always clear for the decision maker. These values, which represent the importance of each combination of criteria, do not give immediately the global importance of the criteria nor the degree of interaction among them.

In fact, from a given fuzzy measure, it is possible to derive some indices or parameters that describe the behavior of the fuzzy measure or, equivalently, that of the Choquet integral that is used to aggregate the criteria. Alternatively, when the fuzzy measure is not completely known, such indices can help the decision maker to assess it. This corresponds to the inverse problem of identifying the weights from parametric specifications on criteria, see [14].

The aim of this paper is to present the following behavioral indices: the global importance of criteria, the interaction among criteria, the influence of the criteria on the aggregation, the tolerance of the decision maker, and the dispersion of the fuzzy measure.

The outline of this paper is as follows. In Section 2 we recall the definition of the discrete Choquet integral and some of its particular cases. Sections 3, 4, and 5 are respectively devoted to the importance indices, interaction indices, and influence indices. Sections 6 and 7 deal with the tolerance of the decision maker by means of the concepts of conjunction and disjunction degrees as well as the veto and favor indices. Section 8 introduces a generalization of the Shannon entropy to measure the dispersion of a fuzzy measure. Finally, in Section 9 we present a practical example demonstrating the use of the parameters presented in this paper.

In order to avoid an heavy notation, cardinality of subsets $S, T, \ldots$ will be denoted whenever possible by the corresponding lower case letters $s, t, \ldots$, otherwise by the standard notation $|S|,|T|, \ldots$ Moreover, we will often omit braces for singletons, e.g., writing $a(i)$, $N \backslash i$ instead of $a(\{i\}), N \backslash\{i\}$. Also, for pairs, we will often write $i j$ instead of $\{i, j\}$, as for example $a(i j)$.

For any subset $S \subseteq N, e_{S}$ will denote the characteristic vector of $S$ in $\{0,1\}^{n}$, i.e., the vector of $\{0,1\}^{n}$ whose $i$-th component is 1 if and only if $i \in S$.

Finally, $\wedge$ and $\vee$ will denote the minimum and maximum operations, respectively. 


\section{The Choquet integral and its particular cases}

The use of the Choquet integral has been proposed by many authors as an adequate substitute to the weighted arithmetic mean to aggregate interacting criteria, see e.g. [6, 12]. In the weighted arithmetic mean model, each criterion $i \in N$ is given a weight $\omega_{i} \in[0,1]$ representing the importance of this criterion in the decision. In the Choquet integral model, where criteria can be dependent, a fuzzy measure [23] is used to define a weight on each combination of criteria, thus making it possible to model the interaction existing among criteria.

Definition 2.1 A fuzzy measure on $N$ is a set function $v: 2^{N} \rightarrow[0,1]$ satisfying the following conditions:

i) $v(\emptyset)=0, v(N)=1$,

ii) $S \subseteq T \Rightarrow v(S) \leq v(T)$.

The set of all fuzzy measures on $N$ will be denoted by $\mathcal{F}_{N}$ as we continue. Moreover, for any fuzzy measure $v$ on $N$ and any permutation $\pi$ on $N, \pi v$ will denote the fuzzy measure on $N$ defined by $\pi v(\pi(S))=v(S)$ for all $S \subseteq N$, where $\pi(S)=\{\pi(i) \mid i \in S\}$.

For any $S \subseteq N, v(S)$ can be interpreted as the weight of importance of the combination $S$ of criteria, or better, its importance or power to make the decision alone (without the remaining criteria).

The concept of Choquet integral was first introduced in capacity theory [1]. Its use as a (fuzzy) integral with respect to a fuzzy measure was then proposed by Murofushi and Sugeno $[17,18]$.

Definition 2.2 Let $v \in \mathcal{F}_{N}$. The Choquet integral of $x: N \rightarrow \mathbb{R}$ with respect to $v$ is defined by

$$
\mathcal{C}_{v}(x):=\sum_{i=1}^{n} x_{(i)}\left[v\left(A_{(i)}\right)-v\left(A_{(i+1)}\right)\right]
$$

where $(\cdot)$ indicates a permutation on $N$ such that $x_{(1)} \leq \ldots \leq x_{(n)}$. Also $A_{(i)}=\{(i), \ldots,(n)\}$, and $A_{(n+1)}=\emptyset$.

Thus defined, the Choquet integral has very good properties for aggregation (see e.g. Grabisch [6]). For instance, it is continuous, non decreasing, comprised between min and max, stable under the same transformations of interval scales in the sense of the theory of measurement, and coincides with the weighted arithmetic mean (discrete Lebesgue integral) as soon as the fuzzy measure is additive. Moreover, in $[11,12]$ the author proposed an axiomatic characterization of the class of all the Choquet integrals with $n$ arguments. The statement is the following.

Theorem 2.1 The operators $M_{v}: \mathbb{R}^{n} \rightarrow \mathbb{R}\left(v \in \mathcal{F}_{N}\right)$ are

- linear w.r.t. the fuzzy measure, that is, there exist $2^{n}$ functions $f_{T}: \mathbb{R}^{n} \rightarrow \mathbb{R}(T \subseteq N)$, such that

$$
M_{v}=\sum_{T \subseteq N} v(T) f_{T}, \quad v \in F_{N}
$$

- non decreasing in each argument, 
- stable for the admissible positive linear transformations, that is,

$$
M_{v}\left(r x+s e_{N}\right)=r M_{v}(x)+s
$$

for all $x \in \mathbb{R}^{n}, r>0, s \in \mathbb{R}$.

- properly weighted by $v$, that is,

$$
M_{v}\left(e_{S}\right)=v(S), \quad S \subseteq N, v \in F_{N},
$$

if and only if $M_{v}=\mathcal{C}_{v}$ for all $v \in \mathcal{F}_{N}$.

The axioms presented in the previous characterization are natural enough in the context of multicriteria decision making. The first one is proposed to keep the aggregation model as simple as possible. The second axiom says that increasing a partial score along any criterion cannot decrease the global score. The third axiom only demands that the aggregated value is stable with respect to any change of scale. Finally, assuming that the partial score scale is embedded in $[0,1]$, the fourth axiom suggests that the weight of importance of any subset $S$ of criteria is defined as the global evaluation of the alternative that completely satisfies criteria $S$ and totally fails to satisfy the others.

The fourth axiom is fundamental. It gives an appropriate definition of the weights of subsets of criteria, interpreting them as global evaluation of particular profiles.

Now, the Möbius transform of a given fuzzy measure $v \in \mathcal{F}_{N}$ is a set function $a: 2^{N} \rightarrow \mathbb{R}$ defined by

$$
a(S)=\sum_{T \subseteq S}(-1)^{s-t} v(T), \quad S \subseteq N .
$$

The transformation is invertible and we have (see e.g. [19])

$$
v(S)=\sum_{T \subseteq S} a(T), \quad S \subseteq N
$$

The Möbius transform enables us to express some functions of $v$ in a simpler form. For example, the Choquet integral is written [12]

$$
\mathcal{C}_{v}(x)=\sum_{T \subseteq N} a(T) \bigwedge_{i \in T} x_{i}, \quad x \in \mathbb{R}^{n} .
$$

We now present some subclasses of Choquet integrals. Any vector $\omega \in[0,1]^{n}$ such that $\sum_{i} \omega_{i}=1$ will be called a weight vector as we continue.

\subsection{The weighted arithmetic mean}

Definition 2.3 For any weight vector $\omega \in[0,1]^{n}$, the weighted arithmetic mean operator $\mathrm{WAM}_{\omega}$ associated to $\omega$ is defined by

$$
\operatorname{WAM}_{\omega}(x)=\sum_{i=1}^{n} \omega_{i} x_{i}
$$


We can easily see that $\mathrm{WAM}_{\omega}$ is a Choquet integral $\mathcal{C}_{v}$ with respect to an additive fuzzy measure:

$$
v(S)=\sum_{i \in S} \omega_{i}, \quad S \subseteq N
$$

The corresponding Möbius representation is given by

$$
\left\{\begin{array}{l}
a(i)=\omega_{i}, \quad \forall i \in N \\
a(S)=0, \quad \forall S \subseteq N \text { such that } s \geq 2 .
\end{array}\right.
$$

Conversely, the weights associated to $\mathrm{WAM}_{\omega}$ are given by

$$
\omega_{i}=v(i)=a(i), \quad i \in N
$$

The class of weighted arithmetic mean $\mathrm{WAM}_{\omega}$ includes two important special cases, namely:

- the arithmetic mean

$$
\operatorname{AM}(x)=\frac{1}{n} \sum_{i=1}^{n} x_{i}
$$

when $\omega_{i}=1 / n$ for all $i \in N$. In this case, we have $v(S)=s / n$ for all $S \subseteq N$ and $a(i)=1 / n$ for all $i \in N$.

- the $k$-th projection

$$
\mathrm{P}_{k}(x)=x_{k},
$$

when $\omega_{k}=1$ for some $k \in N$. In this case, we have $v(S)=1$ if $S \ni k$ and 0 otherwise.

Moreover, we have $a(i)=1$ if $i=k$ and 0 otherwise.

\subsection{The ordered weighted averaging operator}

Yager [25] defined in 1988 the ordered weighted averaging operators (OWA) as follows.

Definition 2.4 For any weight vector $\omega \in[0,1]^{n}$, the ordered weighted averaging operator $\mathrm{OWA}_{\omega}$ associated to $\omega$ is defined by

$$
\operatorname{OWA}_{\omega}(x)=\sum_{i=1}^{n} \omega_{i} x_{(i)}
$$

with the usual convention that $x_{(1)} \leq \cdots \leq x_{(n)}$.

The following result, due to Grabisch [5], shows that any OWA operator is a Choquet integral w.r.t. a fuzzy measure that depends only on the cardinality of subsets.

Proposition 2.1 Let $v \in \mathcal{F}_{N}$. Then the following assertions are equivalent.

i) For any $S, S^{\prime} \subseteq N$ such that $|S|=\left|S^{\prime}\right|$, we have $v(S)=v\left(S^{\prime}\right)$.

ii) There exists a weight vector $\omega$ such that $\mathcal{C}_{v}=\mathrm{OWA}_{\omega}$.

iii) $\mathcal{C}_{v}$ is a symmetric function. 
The fuzzy measure $v$ associated to $\mathrm{OWA}_{\omega}$ is given by

$$
v(S)=\sum_{i=n-s+1}^{n} \omega_{i}, \quad S \subseteq N, \quad S \neq \emptyset
$$

and its Möbius representation by [7, Theorem 1]

$$
a(S)=\sum_{j=0}^{s-1}\left(\begin{array}{c}
s-1 \\
j
\end{array}\right)(-1)^{s-1-j} \omega_{n-j}, \quad S \subseteq N, \quad S \neq \emptyset .
$$

Conversely, the weights associated to $\mathrm{OWA}_{\omega}$ are given by

$$
\omega_{n-s}=v(S \cup i)-v(S)=\sum_{T \subseteq S} a(T \cup i), \quad i \in N, S \subseteq N \backslash i .
$$

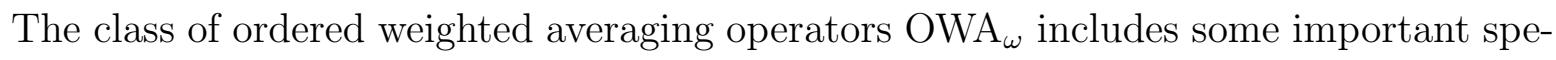
cial cases, namely:

- the arithmetic mean AM when $\omega_{i}=1 / n$ for all $i \in N$.

- the $k$-th order statistic

$$
\mathrm{OS}_{k}(x)=x_{(k)},
$$

when $\omega_{k}=1$ for some $k \in N$. In this case, we have

$$
\begin{aligned}
& v(S)= \begin{cases}1 & \text { if } s \geq n-k+1 \\
0 & \text { otherwise, }\end{cases} \\
& a(S)= \begin{cases}(-1)^{s-n+k-1}\left(\begin{array}{l}
s-1 \\
n-k
\end{array}\right) & \text { if } s \geq n-k+1 \\
0 & \text { otherwise }\end{cases}
\end{aligned}
$$

- the min operator

$$
\min (x)=\min _{i \in N} x_{i}
$$

when $\omega_{1}=1$. In this case, we have $v(S)=a(S)=1$ if $S=N$ and 0 otherwise.

- the max operator

$$
\max (x)=\max _{i \in N} x_{i}
$$

when $\omega_{n}=1$. In this case, we have $v(S)=1$ and $a(S)=(-1)^{s+1}$ for all $S \neq \emptyset$.

\subsection{The partial minimum and maximum operators}

Definition 2.5 For any non-empty subset $T \subseteq N$, the partial minimum operator $\min _{T}$ and the partial maximum operator $\max _{T}$, associated to $T$, are respectively defined by

$$
\begin{aligned}
\min _{T}(x) & =\min _{i \in T} x_{i} \\
\max _{T}(x) & =\min _{i \in T} x_{i} .
\end{aligned}
$$


For the operator $\min _{T}$, we have

$$
\begin{aligned}
& v(S)= \begin{cases}1, & \text { if } S \supseteq T, \\
0, & \text { else },\end{cases} \\
& a(S)= \begin{cases}1, & \text { if } S=T, \\
0, & \text { else. }\end{cases}
\end{aligned}
$$

For the operator $\max _{T}$, we have

$$
\begin{aligned}
& v(S)= \begin{cases}1, & \text { if } S \cap T \neq \emptyset, \\
0, & \text { else, }\end{cases} \\
& a(S)= \begin{cases}(-1)^{s+1}, & \text { if } \emptyset \neq S \subseteq T, \\
0, & \text { else }\end{cases}
\end{aligned}
$$

\section{Importance indices}

The overall importance of a criterion $i \in N$ into a decision problem is not solely determined by the number $v(i)$, but also by all $v(T)$ such that $T \ni i$. Indeed, we may have $v(i)=0$, suggesting that element $i$ is unimportant, but it may happen that for many subsets $T \subseteq$ $N \backslash i, v(T \cup i)$ is much greater than $v(T)$, suggesting that $i$ is actually an important element in the decision.

Shapley [22] has proposed in 1953 a definition of a coefficient of importance, based on a set of reasonable axioms. The importance index or Shapley value of criterion $i$ with respect to $v$ is defined by:

$$
\phi(v, i):=\sum_{T \subseteq N \backslash i} \frac{(n-t-1) ! t !}{n !}[v(T \cup i)-v(T)] .
$$

The Shapley value is a fundamental concept in game theory expressing a power index. Its use in multicriteria decision making was proposed in 1992 by Murofushi [15].

Thus defined, it can be interpreted as a weighted average value of the marginal contribution $v(T \cup i)-v(T)$ of element $i$ alone in all combinations. To make this clearer, it is informative to rewrite the index as follows:

$$
\phi(v, i)=\frac{1}{n} \sum_{t=0}^{n-1} \frac{1}{\left(\begin{array}{c}
n-1 \\
t
\end{array}\right)} \sum_{\substack{T \subseteq N \backslash \backslash i \\
|T|=t}}[v(T \cup i)-v(T)] .
$$

Thus, the average value of $v(T \cup i)-v(T)$ is computed first over all the subsets of the same size $t$ and then over all the possible sizes.

It is worth noting that the Shapley value fulfills the following properties:

$$
\phi(v, i) \geq 0, \quad i \in N
$$

and

$$
\sum_{i=1}^{n} \phi(v, i)=1
$$

Note also that, when $v$ is additive, we clearly have $v(T \cup i)-v(T)=v(i)$ for all $i \in N$ and all $T \subseteq N \backslash i$, and hence

$$
\phi(v, i)=v(i), \quad i \in N .
$$


If $v$ is non-additive then some criteria are dependent and (2) generally does not hold anymore. This shows that it is reasonable to search for a coefficient of overall importance for each criterion.

In terms of the Möbius representation, the Shapley value takes a very simple form [22]:

$$
\phi(v, i)=\sum_{T \ni i} \frac{1}{t} a(T) .
$$

The best known axiomatic supporting the Shapley value is given in the following theorem, see e.g. [22].

Theorem 3.1 The numbers $\psi(v, i)\left(i \in N, v \in \mathcal{F}_{N}\right)$ :

- are linear w.r.t. the fuzzy measure, that is, there exist real constants $p_{T}^{i}(T \subseteq N)$ such that

$$
\psi(v, i)=\sum_{T \subseteq N} p_{T}^{i} v(T), \quad i \in N, v \in \mathcal{F}_{N} .
$$

- are symmetric, that is, for any permutation $\pi$ on $N$, we have

$$
\psi(v, i)=\psi(\pi v, \pi(i)), \quad i \in N, v \in \mathcal{F}_{N} .
$$

- fulfill the "null criterion" axiom, that is, for any $i \in N$ and any $v \in \mathcal{F}_{N}$,

$$
v(T \cup i)=v(T) \quad \forall T \subseteq N \backslash i \quad \Rightarrow \quad \psi(v, i)=0 .
$$

- fulfill the "efficiency" axiom, that is,

$$
\sum_{i=1}^{n} \psi(v, i)=1, \quad v \in \mathcal{F}_{N}
$$

if and only if $\psi(v, i)=\phi(v, i)$ for all $i \in N$ and all $v \in \mathcal{F}_{N}$.

Let us comment on the axioms presented in this characterization. As for the Choquet integral (cf. Theorem 2.1), we ask the importance indices to be linear w.r.t. the corresponding fuzzy measure. Next, the symmetry axiom demands that the indices are independent of the name (label) given to each criterion. The third axiom, which is quite natural, says that when a criterion does not contribute in the decision problem then it has a zero global importance. The last axiom naturally acts as a normalization property.

Table 1 gives the importance indices for fuzzy measures corresponding to particular Choquet integrals.

\section{Interaction indices}

Another interesting concept is that of interaction among criteria. We have seen that when the fuzzy measure is not additive then some criteria interact. Of course, it would be interesting to appraise the degree of interaction among any subset of criteria. 


\begin{tabular}{|c|c|c|}
\hline Fuzzy measure $v$ & & $\phi(v, i)$ \\
\hline$v_{\mathrm{WAM}_{\omega}}$ & & $\omega_{i}$ \\
\hline$v_{\mathrm{OWA}_{\omega}}$ & & $1 / n$ \\
\hline$v_{\min _{T}}$ & $\left\{\begin{array}{l}1 / t \\
0\end{array}\right.$ & $\begin{array}{l}\text { if } i \in T \\
\text { otherwise }\end{array}$ \\
\hline$v_{\max _{T}}$ & $\left\{\begin{array}{l}1 / t \\
0\end{array}\right.$ & $\begin{array}{l}\text { if } i \in T \\
\text { otherwise }\end{array}$ \\
\hline
\end{tabular}

Table 1: Importance indices for various fuzzy measures

Consider first a pair $\{i, j\} \subseteq N$ of criteria. It may happen that $v(i)$ and $v(j)$ are small and at the same time $v(i j)$ is large. Clearly, the number $\phi(v, i)$ merely measures the average contribution that criterion $i$ brings to all possible combinations, but it does not explain why criterion $i$ may have a large importance. In other words, it gives no information on the interaction phenomena existing among criteria.

Suppose that $i$ and $j$ are positively correlated or competitive (resp. negatively correlated or complementary). Then the marginal contribution of $j$ to every combination of criteria that contains $i$ should be strictly less than (resp. greater than) the marginal contribution of $j$ to the same combination when $i$ is excluded. Thus, depending on whether the correlation between $i$ and $j$ is positive or negative, the expression

$$
v(T \cup i j)-v(T \cup i)-v(T \cup j)+v(T)
$$

is $\leq 0$ or $\geq 0$ for all $T \subseteq N \backslash i j$, respectively. We call this expression the marginal interaction between $i$ and $j$, conditioned to the presence of elements of the combination $T \subseteq N \backslash i j$. Now, an interaction index for $\{i, j\}$ is given by an average value of this marginal interaction. Murofushi and Soneda [16] proposed in 1993 to calculate this average value as for the Shapley value. Setting

$$
\left(\Delta_{i j} v\right)(T):=v(T \cup i j)-v(T \cup i)-v(T \cup j)+v(T)
$$

the interaction index of criteria $i$ and $j$ related to $v$ is then defined by

$$
I(v, i j):=\sum_{T \subseteq N \backslash i j} \frac{(n-t-2) ! t !}{(n-1) !}\left(\Delta_{i j} v\right)(T) .
$$

We immediately see that this index is negative as soon as $i$ and $j$ are positively correlated or competitive. Similarly, it is positive when $i$ and $j$ are negatively correlated or complementary. Moreover, it has been shown in [8] that $I(v, i j) \in[-1,1]$ for all $i, j \in N$.

The interaction index among a combination $S$ of criteria was introduced by Grabisch [8] as a natural extension of the case $s=2$. It was also axiomatized very recently by Grabisch 
and Roubens [10]. The interaction index of $S(s \geq 2)$ related to $v$, is defined by

$$
I(v, S):=\sum_{T \subseteq N \backslash S} \frac{(n-t-s) ! t !}{(n-s+1) !}\left(\Delta_{S} v\right)(T),
$$

where

$$
\left(\Delta_{S} v\right)(T):=\sum_{L \subseteq S}(-1)^{s-l} v(L \cup T) .
$$

In terms of the Möbius representation, it is written [8]

$$
I(v, S)=\sum_{T \supseteq S} \frac{1}{t-s+1} a(T), \quad S \subseteq N .
$$

Viewed as a set function, it coincides on singletons with the Shapley value (1). Moreover, it was proved in [9] that the transformation (3) is invertible and its inverse is written:

$$
a(S)=\sum_{T \supseteq S} B_{t-s} I(v, T), \quad S \subseteq N
$$

where $B_{n}$ is the $n$-th Bernoulli number, that is, the $n$-th element of the numerical sequence $\left\{B_{n}\right\}_{n \in \mathbb{N}}$ defined recursively by

$$
\left\{\begin{array}{l}
B_{0}=1 \\
\sum_{k=0}^{n}\left(\begin{array}{c}
n+1 \\
k
\end{array}\right) B_{k}=0, \quad n \in \mathbb{N} \backslash\{0\} .
\end{array}\right.
$$

Table 2 gives the interaction indices for fuzzy measures corresponding to particular Choquet integrals, see $[7,12]$. For $v_{\mathrm{OS}_{k}}$, we have used the convention that $\left(\begin{array}{l}p \\ q\end{array}\right)=0$ whenever $p<q$ or $q<0$.

\section{$5 \quad$ Influence indices}

In Section 3 we have presented the concept of overall importance of each criterion. Now, it would be natural to appraise the global importance (or influence) of any combination of criteria on the aggregation process.

In [13] the author proposed the following definition of the influence of criteria on the Choquet integral. Given a subset $S \subseteq N$ of criteria, the influence of $S$ on $\mathcal{C}_{v}$ is defined as the average amplitude of the range of $\mathcal{C}_{v}$ that criteria $S$ may control when assigning partial scores to the criteria not in $S$ at random.

Definition 5.1 The influence of $S \subseteq N$ on $\mathcal{C}_{v}:[0,1]^{n} \rightarrow \mathbb{R}$ is defined by

$$
I\left(\mathcal{C}_{v}, S\right):=\int_{0}^{1} \cdots \int_{0}^{1}\left[\lim _{\substack{x_{j} \rightarrow 1 \\ j \in S}} \mathcal{C}_{v}(x)-\lim _{\substack{x_{j} \rightarrow 0 \\ j \in S}} \mathcal{C}_{v}(x)\right] d x_{i_{1}} \cdots d x_{i_{n-s}}
$$

where $N \backslash S=\left\{i_{1}, \ldots, i_{n-s}\right\}$. 


\begin{tabular}{|c|c|}
\hline Fuzzy measure $v$ & $I(v, S)$ for $s \geq 2$ \\
\hline$v_{\text {WAM }_{\omega}}$ & 0 \\
\hline$v_{\mathrm{OWA}_{\omega}}$ & $\frac{1}{n-s+1} \sum_{j=0}^{s-2}\left(\begin{array}{c}s-2 \\
j\end{array}\right)(-1)^{s-j}\left(\omega_{s-j-1}-\omega_{n-j}\right)$ \\
\hline$v_{\mathrm{OS}_{k}}$ & $\frac{(-1)^{k+1}}{n-s+1}\left[\left(\begin{array}{c}s-2 \\
n-k\end{array}\right)(-1)^{n-s}+\left(\begin{array}{c}s-2 \\
s-k-1\end{array}\right)\right]$ \\
\hline$v_{\min _{T}}$ & $\begin{cases}\frac{1}{t-s+1} & \text { if } T \supseteq S \\
0 & \text { otherwise }\end{cases}$ \\
\hline$v_{\max _{T}}$ & $\begin{cases}\frac{(-1)^{s+1}}{t-s+1} & \text { if } T \supseteq S \\
0 & \text { otherwise }\end{cases}$ \\
\hline
\end{tabular}

Table 2: Interaction indices for various fuzzy measures

From this definition, the following formulas can be obtained [13]:

$$
\begin{aligned}
I\left(\mathcal{C}_{v}, S\right) & =\sum_{T \subseteq N \backslash S} \frac{(n-t-s) ! t !}{(n-s+1) !}[v(T \cup S)-v(T)], \quad S \subseteq N, \\
I\left(\mathcal{C}_{v}, S\right) & =\sum_{\substack{T \subseteq N \\
T \cap S \neq \emptyset}} a(T) \frac{1}{|T \backslash S|+1}, \quad S \subseteq N .
\end{aligned}
$$

Moreover, we clearly have $I\left(\mathcal{C}_{v}, S\right) \in[0,1]$ for all $S \subseteq N$, and we can see that the closer $I\left(\mathcal{C}_{v}, S\right)$ is to 1 , the more the subset $S$ has influence on the aggregation.

As for the interaction index, the influence index coincides on singletons with the Shapley value (1). However, the transformations (4) and (5), although they are linear, are not invertible.

Table 3 gives the influence indices for particular Choquet integrals, see [13].

\section{Conjunction and disjunction degrees}

Consider the cube $[0,1]^{n}$ as a probability space with uniform distribution. Then the expected value of $\mathcal{C}_{v}(x)$, that is,

$$
\mathrm{E}\left(\mathcal{C}_{v}\right)=\int_{[0,1]^{n}} \mathcal{C}_{v}(x) d x
$$

represents the average value of the Choquet integral $\mathcal{C}_{v}$ over $[0,1]^{n}$. This expression gives the average position of $\mathcal{C}_{v}$ within the interval $[0,1]$. 


\begin{tabular}{|c|c|c|}
\hline Choquet integral $\mathcal{C}_{v}$ & \multicolumn{2}{|c|}{$I\left(\mathcal{C}_{v}, S\right)$} \\
\hline $\mathrm{WAM}_{\omega}$ & $\sum_{i \in S}$ & \\
\hline $\mathrm{OWA}_{\omega}$ & $\sum_{i=1}^{n} \omega_{i} \frac{i \wedge s \wedge(n-i}{n}$ & $\frac{+1) \wedge(n-s+1)}{-s+1}$ \\
\hline $\mathrm{OS}_{k}$ & $\frac{k \wedge s \wedge(n-k+}{n-s}$ & $\frac{1) \wedge(n-s+1)}{+1}$ \\
\hline $\min _{T}$ & $\left\{\begin{array}{l}\frac{1}{|T \backslash S|+1} \\
0\end{array}\right.$ & $\begin{array}{l}\text { if } T \cap S \neq \emptyset \\
\text { otherwise }\end{array}$ \\
\hline $\max _{T}$ & $\left\{\begin{array}{l}\frac{1}{|T \backslash S|+1} \\
0\end{array}\right.$ & $\begin{array}{l}\text { if } T \cap S \neq \emptyset \\
\text { otherwise }\end{array}$ \\
\hline
\end{tabular}

Table 3: Influence indices for various Choquet integrals

Since the Choquet integral is always internal to the set of its arguments, that is

$$
\min x_{i} \leq \mathcal{C}_{v}(x) \leq \max x_{i}, \quad x \in[0,1]^{n},
$$

from (6) it follows that

$$
\mathrm{E}(\min ) \leq \mathrm{E}\left(\mathcal{C}_{v}\right) \leq \mathrm{E}(\max ), \quad v \in \mathcal{F}_{N}
$$

The relative position of $\mathrm{E}\left(\mathcal{C}_{v}\right)$ with respect to the lower bound of the interval [E(min), $\mathrm{E}(\max )$ ] is called the conjunction degree or the degree of andness of $\mathcal{C}_{v}$. It represents the degree to which the average value of $\mathcal{C}_{v}$ is close to that of min.

Definition 6.1 The degree of andness of $\mathcal{C}_{v}$ is defined by

$$
\operatorname{andness}\left(\mathcal{C}_{v}\right):=\frac{\mathrm{E}(\max )-\mathrm{E}\left(\mathcal{C}_{v}\right)}{\mathrm{E}(\max )-\mathrm{E}(\min )}
$$

Similarly, the relative position of $\mathrm{E}\left(\mathcal{C}_{v}\right)$ with respect to $\mathrm{E}(\max )$ is called the disjunction degree or the degree of orness of $\mathcal{C}_{v}$.

Definition 6.2 The degree of orness of $\mathcal{C}_{v}$, is defined by

$$
\operatorname{orness}\left(\mathcal{C}_{v}\right):=\frac{\mathrm{E}\left(\mathcal{C}_{v}\right)-\mathrm{E}(\min )}{\mathrm{E}(\max )-\mathrm{E}(\min )}
$$


An immediate consequence of these definitions is that

$$
\operatorname{andness}\left(\mathcal{C}_{v}\right)+\operatorname{orness}\left(\mathcal{C}_{v}\right)=1 \text {. }
$$

Moreover, we have andness $\left(\mathcal{C}_{v}\right)$, orness $\left(\mathcal{C}_{v}\right) \in[0,1]$.

These two concepts have been introduced as early as 1974 by Dujmovic $[3,4]$ in the particular case of power means. Here we have adapted his definitions to the Choquet integral.

The degree of orness is a measure of the tolerance of the decision maker. Indeed, tolerant decision makers can accept that only some criteria are satisfied. This corresponds to a disjunctive behavior $\left(\operatorname{orness}\left(\mathcal{C}_{v}\right)>0.5\right)$, whose extreme example is max. On the other hand, intolerant decision makers demand that most criteria are satisfied. This corresponds to a conjunctive behavior (orness $\left(\mathcal{C}_{v}\right)<0.5$ ), whose extreme example is min. When orness $\left(\mathcal{C}_{v}\right)=$ 0.5 the decision maker is medium (neither tolerant nor intolerant).

Using the identity

$$
\int_{[0,1]^{n}} \bigwedge_{i \in S} x_{i} d x=\frac{1}{s+1}, \quad S \subseteq N
$$

(see [9]) and the expression of $\mathcal{C}_{v}$ in terms of the Möbius representation, we have the following result.

Proposition 6.1 For any $v \in \mathcal{F}_{N}$, we have

$$
\operatorname{orness}\left(\mathcal{C}_{v}\right)=\frac{1}{n-1} \sum_{T \subseteq N} \frac{n-t}{t+1} a(T)
$$

where a is the Möbius representation of $v$.

In terms of the fuzzy measure $v$ the degree of orness takes the following form, see [11].

Proposition 6.2 For any $v \in \mathcal{F}_{N}$, we have

$$
\operatorname{orness}\left(\mathcal{C}_{v}\right)=\frac{1}{n-1} \sum_{T \varsubsetneqq N} \frac{(n-t) ! t !}{n !} v(T),
$$

that is,

$$
\operatorname{orness}\left(\mathcal{C}_{v}\right)=\frac{1}{n-1} \sum_{t=1}^{n-1} \frac{1}{\left(\begin{array}{l}
n \\
t
\end{array}\right)} \sum_{\substack{T \subseteq N \\
|T|=t}} v(T) .
$$

Corollary 6.1 Let $v \in \mathcal{F}_{N}$. Then

$$
\begin{aligned}
& \operatorname{orness}\left(\mathcal{C}_{v}\right)=0 \quad \Leftrightarrow \quad \mathcal{C}_{v}=\min \\
& \operatorname{orness}\left(\mathcal{C}_{v}\right)=1 \quad \Leftrightarrow \quad \mathcal{C}_{v}=\max
\end{aligned}
$$

The concept of orness was defined independently by Yager [25] in the particular case of OWA operators, see also Yager [26]. His definition is based on the use of the so-called regular increasing monotone quantifiers, that is, increasing functions $Q:[0,1] \rightarrow[0,1]$, with $Q(0)=0$ and $Q(1)=1$, which represent linguistic quantifiers such as all, most, many, 
at least $k$. For any $k \in N, Q(k / n)$ indicates the lowest global evaluation of an alternative that fully satisfies $k$ criteria:

$$
Q(k / n)=\operatorname{OWA}_{\omega}\left(e_{\{1, \ldots, k\}}\right)=\sum_{i=1}^{k} \omega_{n-i+1} .
$$

Yager then defined

$$
\operatorname{orness}\left(\mathrm{OWA}_{\omega}\right)=\frac{1}{n-1} \sum_{k=1}^{n-1} Q(k / n)=\frac{1}{n-1} \sum_{k=1}^{n}(k-1) \omega_{k} .
$$

These definitions can be adapted to the more general case of the Choquet integrals. The average value of the lowest global evaluation over all the alternatives that fully satisfy $k$ criteria is given by

$$
Q(k / n)=\frac{1}{\left(\begin{array}{l}
n \\
k
\end{array}\right)} \sum_{\substack{K \subseteq N \\
|K|=k}} \mathcal{C}_{v}\left(e_{K}\right), \quad k \in N .
$$

The orness of $\mathcal{C}_{v}$ can then be defined by

$$
\operatorname{orness}\left(\mathcal{C}_{v}\right)=\frac{1}{n-1} \sum_{k=1}^{n-1} Q(k / n),
$$

and we retrieve (7).

\begin{tabular}{|c|c|c|}
\hline Choquet integral $\mathcal{C}_{v}$ & $\operatorname{andness}\left(\mathcal{C}_{v}\right)$ & $\operatorname{orness}\left(\mathcal{C}_{v}\right)$ \\
\hline WAM $_{\omega}$ & $\frac{1}{2}$ & $\frac{1}{2}$ \\
OWA $_{\omega}$ & $\frac{1}{n-1} \sum_{i=1}^{n}(n-i) \omega_{i}$ & $\frac{1}{n-1} \sum_{i=1}^{n}(i-1) \omega_{i}$ \\
OS $_{k}$ & $\frac{n-k}{n-1}$ & $\frac{k-1}{n-1}$ \\
$\min _{T}$ & $\frac{n t-1}{(n-1)(t+1)}$ & $\frac{n-t}{(n-1)(t+1)}$ \\
$\max _{T}$ & $\frac{n-t}{(n-1)(t+1)}$ & $\frac{n t-1}{(n-1)(t+1)}$ \\
\hline
\end{tabular}

Table 4: Degrees of andness and orness for various Choquet integrals

Table 4 gives the degrees of andness and orness for particular Choquet integrals.

It can also be interesting to appraise the dispersion of $\mathcal{C}_{v}$ around its average value by calculating its variance, that is,

$$
\sigma^{2}\left(\mathcal{C}_{v}\right)=\int_{[0,1]^{n}}\left[\mathcal{C}_{v}(x)-\mathrm{E}\left(\mathcal{C}_{v}\right)\right]^{2} d x .
$$


In terms of the Möbius representation, this expression is written

$$
\sigma^{2}\left(\mathcal{C}_{v}\right)=\sum_{S, T \subseteq N} a(S) a(T) \frac{s+t-|S \cup T|}{(s+1)(t+1)(|S \cup T|+2)} .
$$

For example,

$$
\begin{aligned}
\sigma^{2}\left(\mathrm{WAM}_{\omega}\right) & =\frac{1}{12} \sum_{i=1}^{n} \omega_{i}^{2} \\
\sigma^{2}\left(\min _{T}\right) & =\frac{t}{(t+1)^{2}(t+2)} \\
\sigma^{2}\left(\max _{T}\right) & =\frac{t}{(t+1)^{2}(t+2)}
\end{aligned}
$$

\section{$7 \quad$ Veto and favor effects}

An interesting behavioral phenomenon in aggregation is the veto effect, and its counterpart, the favor effect. A criterion $k \in N$ is said to be a veto or a blocker for $\mathcal{C}_{v}$ if its non satisfaction entails necessarily a low global score. Formally, $k$ is a veto for $\mathcal{C}_{v}$ if

$$
\mathcal{C}_{v}(x) \leq x_{k}, \quad x \in[0,1]^{n} .
$$

Similarly, the criterion $k$ is a favor or a pusher for $\mathcal{C}_{v}$ if its satisfaction entails necessarily a high global score:

$$
\mathcal{C}_{v}(x) \geq x_{k}, \quad x \in[0,1]^{n} .
$$

The concepts of veto and favor were already proposed by Dubois and Koning [2] in the context of social choice functions, where "favor" was called "dictator".

A consequence of the definition is that no aggregation operator can model simultaneously a veto on a criterion and a favor on another one. Indeed it is not possible to have

$$
x_{i} \leq \mathcal{C}_{v}(x) \leq x_{j} \text { for all } x \in[0,1]^{n} .
$$

Note that if the decision maker considers that a given criterion must absolutely be satisfied (veto criterion), then he/she is conjunctive oriented. Indeed, by (8) we have $\operatorname{orness}\left(\mathcal{C}_{v}\right) \leq 0.5$, which is sufficient. Similarly, if the decision maker considers that a given criterion is sufficient to be satisfied (favor criteria) then he/she is disjunctive oriented. By (9) we have orness $\left(\mathcal{C}_{v}\right) \geq 0.5$.

The following two results can be easily proved [11].

Proposition 7.1 Let $k \in N$ and $v \in \mathcal{F}_{N}$. Then the following assertions are equivalent:

i) $k$ is a veto for $\mathcal{C}_{v}$

ii) $v(T)=0$ whenever $T \not \supset k$ (or, equivalently, $v(N \backslash k)=0$ )

iii) $\exists \lambda \in\left[0,1\left[\right.\right.$ such that $\forall x \in[0,1]^{n}$, with $x_{k} \leq \lambda$, we have $\mathcal{C}_{v}(x) \leq \lambda$

Proposition 7.2 Let $k \in N$ and $v \in \mathcal{F}_{N}$. Then the following assertions are equivalent:

i) $k$ is a favor for $\mathcal{C}_{v}$

ii) $v(T)=1$ whenever $T \ni k$ (or, equivalently, $v(k)=1$ )

iii) $\exists \lambda \in] 0,1]$ such that $\forall x \in[0,1]^{n}$, with $x_{k} \geq \lambda$, we have $\mathcal{C}_{v}(x) \geq \lambda$ 
It seems reasonable to define indices that measure the degree of veto or favor of a given criterion. A natural definition of a degree of veto (resp. favor) consists in considering the probability

$$
\operatorname{Pr}\left[\mathcal{C}_{v}(x) \leq x_{k}\right] \quad\left(\text { resp. } \operatorname{Pr}\left[\mathcal{C}_{v}(x) \geq x_{k}\right]\right)
$$

where $x \in[0,1]^{n}$ is a multi-dimensional random variable uniformly distributed. Unfortunately, such an index does not depend always continuously on $v$. For example, one can easily show [11] that

$$
\operatorname{Pr}\left[\operatorname{WAM}_{\omega}(x) \leq x_{k}\right]= \begin{cases}1, & \text { if } \omega_{k}=1 \\ 1 / 2, & \text { otherwise }\end{cases}
$$

As for power and interaction indices, we search for veto and favor indices which are linear with respect to the fuzzy measure. In [11] the author introduced the following indices, based on an axiomatic characterization:

$$
\begin{aligned}
\operatorname{veto}\left(\mathcal{C}_{v}, i\right) & :=1-\frac{1}{n-1} \sum_{T \subseteq N \backslash i} \frac{(n-t-1) ! t !}{(n-1) !} v(T), \quad i \in N, \\
\operatorname{favor}\left(\mathcal{C}_{v}, i\right) & :=\frac{1}{n-1} \sum_{T \subseteq N \backslash i} \frac{(n-t-1) ! t !}{(n-1) !} v(T \cup i)-\frac{1}{n-1}, \quad i \in N .
\end{aligned}
$$

In terms of the Möbius representation of $v$, these indices are written:

$$
\begin{aligned}
\operatorname{veto}\left(\mathcal{C}_{v}, i\right) & =1-\frac{n}{n-1} \sum_{T \subseteq N \backslash i} \frac{1}{t+1} a(T), \quad i \in N, \\
\operatorname{favor}\left(\mathcal{C}_{v}, i\right) & =\frac{n}{n-1} \sum_{T \subseteq N \backslash i} \frac{1}{t+1}[a(T \cup i)+a(T)]-\frac{1}{n-1}, \quad i \in N,
\end{aligned}
$$

The axiomatic that supports these indices is given in the following result.

Theorem 7.1 The numbers $\psi\left(\mathcal{C}_{v}, i\right)\left(i \in N, v \in \mathcal{F}_{N}\right)$ :

- are linear w.r.t. the fuzzy measure, that is, there exist real constants $p_{T}^{i}(T \subseteq N)$ such that

$$
\psi\left(\mathcal{C}_{v}, i\right)=\sum_{T \subseteq N} p_{T}^{i} v(T), \quad i \in N, v \in \mathcal{F}_{N}
$$

- are symmetric, that is, for any permutation $\pi$ on $N$, we have

$$
\psi\left(\mathcal{C}_{v}, i\right)=\psi\left(\mathcal{C}_{\pi v}, \pi(i)\right), \quad i \in N, v \in \mathcal{F}_{N}
$$

- fulfill the "boundary" axiom, that is, for any $T \subseteq N, T \neq \emptyset$, and any $i \in T$, we have

$$
\psi\left(\min _{T}, i\right)=1, \quad\left(\operatorname{resp} . \psi\left(\max _{T}, i\right)=1\right)
$$

- fulfill the "normalization" axiom, that is, for any $v \in \mathcal{F}_{N}$,

$$
\begin{gathered}
\psi\left(\mathcal{C}_{v}, i\right)=\psi\left(\mathcal{C}_{v}, j\right) \quad \forall i, j \in N \\
\Downarrow \\
\psi\left(\mathcal{C}_{v}, i\right)=\operatorname{andness}\left(\mathcal{C}_{v}\right) \quad\left(r e s p . \operatorname{orness}\left(\mathcal{C}_{v}\right)\right) \quad \forall i \in N .
\end{gathered}
$$


if and only if $\psi\left(\mathcal{C}_{v}, i\right)=\operatorname{veto}\left(\mathcal{C}_{v}, i\right)\left(\right.$ resp. favor $\left.\left(\mathcal{C}_{v}, i\right)\right)$ for all $i \in N$ and all $v \in \mathcal{F}_{N}$.

Let us comment on the axioms presented in this characterization. As for the importance indices, we ask the veto and favor indices to be linear w.r.t. the fuzzy measure and symmetric. Next, the boundary axiom is motivated by the observation that any $i \in T$ is a veto (resp. favor) criterion for $\min _{T}\left(\right.$ resp. $\left.\max _{T}\right)$. Finally, the normalization axiom says that if the degree of veto (resp. favor) does not depend on criteria, then it identifies with the degree of intolerance (resp. tolerance) of the decision maker.

It is easy to observe that $\operatorname{veto}\left(\mathcal{C}_{v}, i\right), \operatorname{favor}\left(\mathcal{C}_{v}, i\right) \in[0,1]$. Furthermore, we have, for any $v \in \mathcal{F}_{N}$,

$$
\begin{aligned}
& \frac{1}{n} \sum_{i=1}^{n} \operatorname{veto}\left(\mathcal{C}_{v}, i\right)=\operatorname{andness}\left(\mathcal{C}_{v}\right), \\
& \frac{1}{n} \sum_{i=1}^{n} \operatorname{favor}\left(\mathcal{C}_{v}, i\right)=\operatorname{orness}\left(\mathcal{C}_{v}\right), \\
& \operatorname{veto}\left(\mathcal{C}_{v}, i\right)+\operatorname{favor}\left(\mathcal{C}_{v}, i\right)=1+\frac{n \phi(v, i)-1}{n-1}, \quad i \in N .
\end{aligned}
$$

Thus defined, we see that $\operatorname{veto}\left(\mathcal{C}_{v}, i\right)$ is more or less the degree to which the decision maker demands that criterion $i$ is satisfied. Similarly, favor $\left(\mathcal{C}_{v}, i\right)$ is the degree to which the decision maker considers that a good score along criterion $i$ is sufficient to be satisfied.

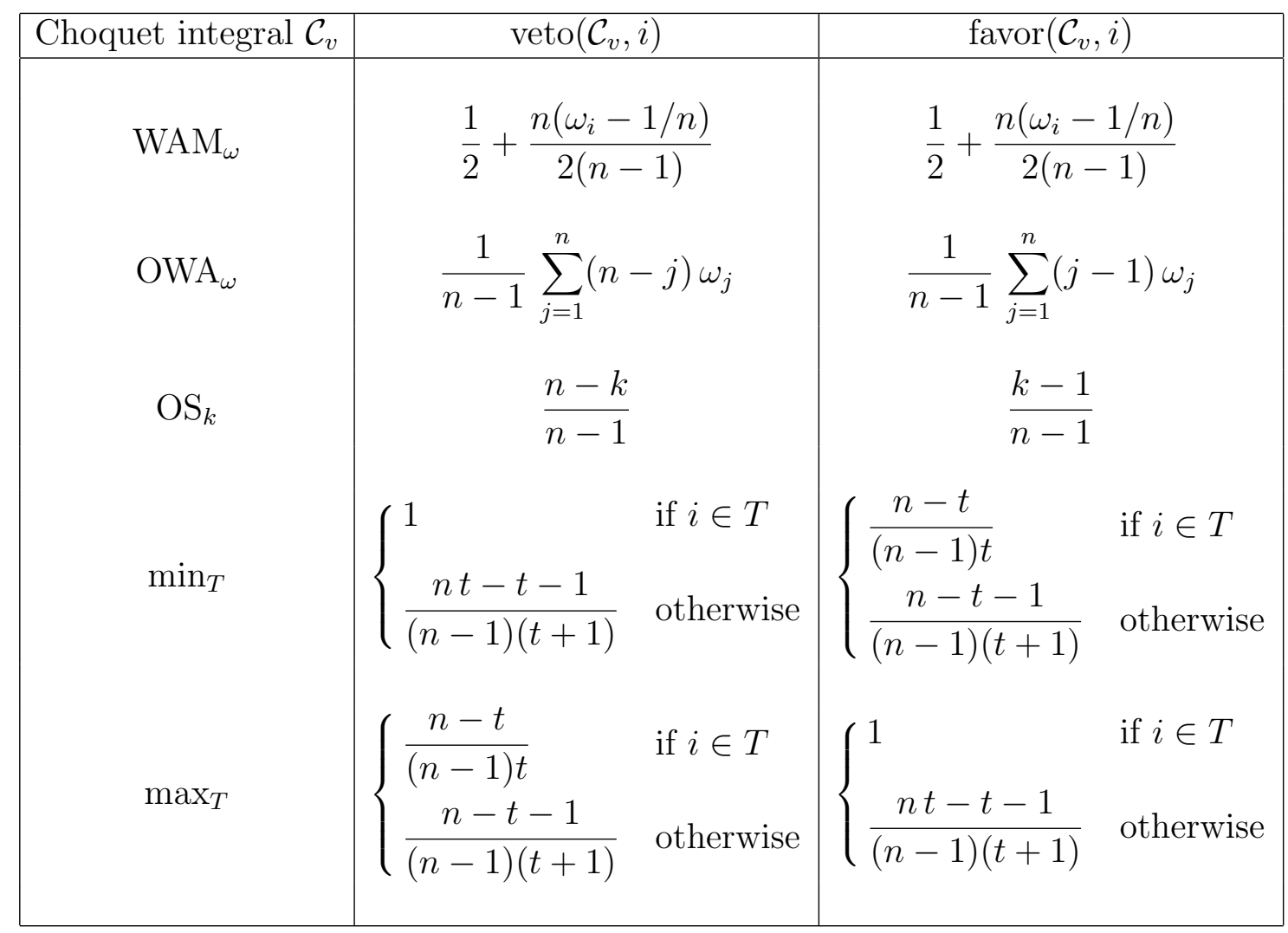

Table 5: Veto and favor indices for various Choquet integrals

Table 5 gives the veto and favor indices for particular Choquet integrals. 


\section{Measure of dispersion}

Consider the weighted arithmetic mean (WAM) as an aggregation operator. It is clear that, in such an aggregation process, the use of the information contained in the arguments $x_{1}, \ldots, x_{n}$ strongly depends upon the weight vector $\omega$. For example, consider two weighted arithmetic means with weights vectors of the form

$$
(1,0, \ldots, 0) \text { and }(1 / n, \ldots, 1 / n)
$$

respectively. We note that these operators are quite different in the sense that the first one focuses the total weight on only one argument (projection on the first argument) whereas the second one distributes the total weight among all the arguments evenly (arithmetic mean).

In order to capture this idea, one can define a measure of dispersion associated to the weight vector of the weighted arithmetic mean $\mathrm{WAM}_{\omega}$ as the Shannon entropy of $\omega$, see $[20,21]$ :

$$
H_{n}(\omega)=-\sum_{i=1}^{n} \omega_{i} \log _{2} \omega_{i}
$$

Such a function enables us to measure how much of the information in the arguments is really used. In a certain sense the more disperse the $\omega$ the more the information contained in the arguments is being used in the aggregation process.

Now, consider the ordered weighted averaging operator (OWA). For this aggregation operator, the measure of dispersion, which should not depend on a reordering of the arguments, should also be given by the Shannon entropy. In fact, Yager [25] proposed explicitely to use this concept as measure of dispersion for the OWA operators.

It is known that $H_{n}(\omega)$ is maximum only when $\omega$ corresponds to the weight vector of the arithmetic mean, see e.g. [24]:

$$
H_{n}(\omega)=\log _{2} n \quad \text { for } \omega=(1 / n, \ldots, 1 / n),
$$

and minimum only when $\omega$ is a binary vector:

$$
H_{n}(\omega)=0 \quad \text { if } \omega_{i}=1 \text { for some } i \in N .
$$

Thus, the measure of dispersion can be normalized into

$$
\operatorname{disp}(\omega)=\frac{1}{\log _{2} n} H_{n}(\omega)=-\sum_{i=1}^{n} \omega_{i} \log _{n} \omega_{i}
$$

so that it ranges in $[0,1]$.

The author $[11, \S 6.2 .4]$ proposed to define the entropy of a fuzzy measure $v \in \mathcal{F}_{N}$ as a measure of dispersion for the Choquet integral $\mathcal{C}_{v}$. This measure should identify with the Shannon entropy when the Choquet integral is either a WAM or an OWA.

On the one hand, comparing

$$
\operatorname{OWA}_{\omega}(x)=\sum_{i=1}^{n} x_{(i)} \omega_{i}
$$

and

$$
\mathcal{C}_{v}(x)=\sum_{i=1}^{n} x_{(i)}\left[v\left(A_{(i)}\right)-v\left(A_{(i+1)}\right)\right]
$$


suggests proposing as measure of dispersion for $\mathcal{C}_{v}$ a sum over $i \in N$ of an average value of

$$
[v(T \cup i)-v(T)] \log _{n}[v(T \cup i)-v(T)], \quad T \subseteq N \backslash i,
$$

that is, an expression of the form

$$
\operatorname{disp}(v)=-\sum_{i=1}^{n} \sum_{T \subseteq N \backslash i} p_{t}[v(T \cup i)-v(T)] \log _{n}[v(T \cup i)-v(T)],
$$

where the coefficients $p_{t}$ are non-negative and such that $\sum_{T \subseteq N \backslash i} p_{t}=1$.

On the other hand, imposing the condition

$$
\mathcal{C}_{v}=\mathrm{OWA}_{\omega} \quad \Rightarrow \quad \operatorname{disp}(v)=\operatorname{disp}(\omega)
$$

determines uniquely the coefficients $p_{t}$, so that the definition proposed is the following.

Definition 8.1 The entropy of a fuzzy measure $v \in \mathcal{F}_{N}$ is defined by

$$
\operatorname{disp}(v):=-\sum_{i=1}^{n} \sum_{T \subseteq N \backslash i} \frac{(n-t-1) ! t !}{n !}[v(T \cup i)-v(T)] \log _{n}[v(T \cup i)-v(T)] .
$$

When the Choquet integral $\mathcal{C}_{v}$ is used as an aggregation operator, this entropy can be interpreted as the degree to which one uses all the information contained in the arguments $x=\left(x_{1}, \ldots, x_{n}\right)$ when calculating the aggregated value $\mathcal{C}_{v}(x)$.

Interestingly enough, its expression (which is of course non linear w.r.t. $v$ ) is very similar to that of the Shapley value (1). Notice also that this new definition has yet to be axiomatically characterized. However, to justify its use, one can show that it fulfills several properties required for an entropy [11], namely:

- $\operatorname{disp}(v)$ is continuous w.r.t. $v$.

- disp is symmetric, that is

$$
\operatorname{disp}(\pi v)=\operatorname{disp}(v)
$$

for any permutation $\pi$ of $N$.

- We have

$$
0 \leq \operatorname{disp}(v) \leq 1
$$

Moreover, $\operatorname{disp}(v)$ is maximum $(=1)$ only when $\mathcal{C}_{v}$ is the arithmetic mean, and minimum $(=0)$ only when $v$ is a binary-valued fuzzy measure: $v(S) \in\{0,1\}$ for all $S \subseteq N$. Note that this latter case occurs if and only if $\mathcal{C}_{v}(x) \in\left\{x_{1}, \ldots, x_{n}\right\}$ (only one piece of information is used in the aggregation).

- We have

$$
\mathcal{C}_{v}=\mathrm{WAM}_{\omega} \text { or } \mathrm{OWA}_{\omega} \Rightarrow \operatorname{disp}(v)=\operatorname{disp}(\omega)
$$

- Let $k \in N$ be a null element for $v$, that is, $v(T \cup k)=v(T)$ for all $T \subseteq N \backslash k$. Then

$$
\operatorname{disp}(v)=\operatorname{disp}\left(v^{N \backslash k}\right)
$$

where $v^{N \backslash k}$ is the restriction of $v$ to $N \backslash k$.

Table 6 gives the entropy of fuzzy measures corresponding to particular Choquet integrals. 


\begin{tabular}{|c|c|}
\hline Fuzzy measure $v$ & $\operatorname{disp}(v)$ \\
\hline$v_{\mathrm{WAM}_{\omega}}$ & $-\sum_{i=1}^{n} \omega_{i} \log _{n} \omega_{i}$ \\
$v_{\mathrm{OWA}_{\omega}}$ & $-\sum_{i=1}^{n} \omega_{i} \log _{n} \omega_{i}$ \\
$v_{\mathrm{OS}_{k}}$ & 0 \\
$v_{\min _{T}}$ & 0 \\
$v_{\max _{T}}$ & 0 \\
\hline
\end{tabular}

Table 6: Entropy of various fuzzy measures

\section{An illustrative example}

In this final section we give an example, borrowed from Grabisch [6]. Let us consider the problem of evaluating students in a high school with respect to three subjects: mathematics $(\mathrm{M})$, physics $(\mathrm{P})$, and literature $(\mathrm{L})$. Usually, this is done by a simple weighted arithmetic mean, whose weights are the coefficients of importance of the different subjects. Suppose that the school is more scientifically than literary oriented, so that weights could be for example 3,3 , and 2 , respectively. Then the weighted arithmetic mean will give the following results for three students $a, b$, and $c$ (marks are given on a scale from 0 to 20):

\begin{tabular}{|c|ccc|c|}
\hline Student & M & P & L & $\begin{array}{c}\text { Global evaluation } \\
\text { (weighted arithmetic mean) }\end{array}$ \\
\hline$a$ & 18 & 16 & 10 & 15.25 \\
$b$ & 10 & 12 & 18 & 12.75 \\
$c$ & 14 & 15 & 15 & 14.625 \\
\hline
\end{tabular}

If the school wants to favor well equilibrated students without weak points then student $c$ should be considered better than student $a$, who has a severe weakness in literature. Unfortunately, no weight vector $\left(\omega_{\mathrm{M}}, \omega_{\mathrm{P}}, \omega_{\mathrm{L}}\right)$ satisfying $\omega_{\mathrm{M}}=\omega_{\mathrm{P}}>\omega_{\mathrm{L}}$ is able to favor student $c$. Indeed, we have:

$$
c \succ a \Longleftrightarrow \omega_{\mathrm{L}}>\omega_{\mathrm{M}} \text {. }
$$

The reason of this problem is that too much importance is given to mathematics and physics, which present some overlap effect since, usually, students good at mathematics are also good at physics (and vice versa), so that the evaluation is overestimated (resp. underestimated) for students good (resp. bad) at mathematics and/or physics. This problem can be solved by using a suitable fuzzy measure $v$ and the Choquet integral.

- Since scientific subjects are more important than literature, the following weights can 
be put on subjects taken individually: $v(\mathrm{M})=v(\mathrm{P})=0.45$ and $v(\mathrm{~L})=0.3$. Note that the initial ratio of weights $(3,3,2)$ is kept unchanged.

- Since mathematics and physics overlap, the weights attributed to the pair $\{M, P\}$ should be less than the sum of the weights of mathematics and physics: $v(\mathrm{MP})=0.5$.

- Since students equally good at scientific subjects and literature must be favored, the weight attributed to the pair $\{\mathrm{L}, \mathrm{M}\}$ should be greater than the sum of individual weights (the same for physics and literature): $v(\mathrm{ML})=v(\mathrm{PL})=0.9$.

- $v(\emptyset)=0$ and $v(\mathrm{MPL})=1$ by definition.

The Möbius representation is then given by

$$
\begin{array}{llll}
a(\emptyset)=0 & a(\mathrm{M})=0.45 & a(\mathrm{MP})=-0.40 & a(\mathrm{MPL})=-0.10 \\
& a(\mathrm{P})=0.45 & a(\mathrm{ML})=0.15 \\
& a(\mathrm{~L})=0.30 & a(\mathrm{PL})=0.15
\end{array}
$$

Applying Choquet integral with the above fuzzy measure leads to the following new global evaluations:

\begin{tabular}{|c|ccc|c|}
\hline Student & $\mathrm{M}$ & $\mathrm{P}$ & $\mathrm{L}$ & $\begin{array}{c}\text { Global evaluation } \\
\text { (Choquet integral) }\end{array}$ \\
\hline$a$ & 18 & 16 & 10 & 13.9 \\
$b$ & 10 & 12 & 18 & 13.6 \\
$c$ & 14 & 15 & 15 & 14.6 \\
\hline
\end{tabular}

The expected result is then obtained. Also remark that student $b$ has still the lowest rank, as requested by the scientific tendency of this high school.

Now, let us turn to a deeper analysis of the orientation of the school or its director. From the fuzzy measure proposed, we obtain the following Shapley value and degrees of veto and favor:

\begin{tabular}{|cccc|}
\cline { 2 - 4 } \multicolumn{1}{c|}{} & $\mathrm{M}$ & $\mathrm{P}$ & $\mathrm{L}$ \\
\hline $\operatorname{veto}\left(\mathcal{C}_{v}, i\right)$ & 0.362 & 0.362 & 0.525 \\
favor $\left(\mathcal{C}_{v}, i\right)$ & 0.575 & 0.575 & 0.6 \\
\hline$\phi(v, i)$ & 0.292 & 0.292 & 0.417 \\
$n * \phi(v, i)$ & 0.875 & 0.875 & 1.25 \\
\hline
\end{tabular}

As we can see, it is convenient to scale the Shapley value by a factor $n$, so that an importance index greater than 1 indicates an attribute more important than the average. Moreover, looking at the veto and favor degrees, we observe that the school seems to favor slightly the students (disjunctive oriented). This is in accordance with the degree of orness

$$
\operatorname{orness}\left(\mathcal{C}_{v}\right)=0.583
$$

We also have $\mathrm{E}\left(\mathcal{C}_{v}\right)=0.542$ and $\sigma\left(\mathcal{C}_{v}\right)=0.180$, showing that the global evaluation is rather dispersed around its typical value 0.542 .

Now, the interaction indices for the pairs of criteria are: 


\begin{tabular}{|c|cc|}
\cline { 2 - 3 } \multicolumn{1}{c|}{} & $\mathrm{P}$ & $\mathrm{L}$ \\
\hline $\mathrm{M}$ & -0.45 & 0.10 \\
$\mathrm{P}$ & & 0.10 \\
\hline
\end{tabular}

Moreover, the influence indices are:

\begin{tabular}{|c|cc|}
\cline { 2 - 3 } \multicolumn{1}{c|}{} & $\mathrm{P}$ & $\mathrm{L}$ \\
\hline $\mathrm{M}$ & 0.60 & 0.725 \\
$\mathrm{P}$ & & 0.725 \\
\hline
\end{tabular}

All these numerical values are also in accordance with the interpretation of the fuzzy measure.

Finally, the dispersion of the fuzzy measure is given by $\operatorname{disp}(v)=0.820$, which is rather satisfactory.

Remark that all these behavioral parameters have been obtained from a given fuzzy measure. In many practical situations, the fuzzy measure is not completely available. We might then fix its values from information on behavioral parameters, see [14].

\section{References}

[1] Choquet G. (1953) Theory of capacities. Annales de l'Institut Fourier 5, 131-295

[2] Dubois D., Koning J.-L. (1991) Social choice axioms for fuzzy set aggregation. Fuzzy Sets and Systems 43, 257-274

[3] Dujmović J.J. (1974) Weighted conjunctive and disjunctive means and their application in system evaluation. Univ. Beograd. Publ. Elektrotechn. Fak., 147-158

[4] Dujmović J.J. (1975) Extended continuous logic and the theory of complex criteria. Univ. Beograd. Publ. Elektrotechn. Fak., 197-216

[5] Grabisch M. (1995) On equivalence classes of fuzzy connectives : the case of fuzzy integrals. IEEE Trans. Fuzzy Systems 3 (1), 96-109

[6] Grabisch M. (1996) The application of fuzzy integrals in multicriteria decision making. European Journal of Operational Research 89, 445-456

[7] Grabisch M. (1997) Alternative representations of OWA operators. In: The Ordered Weighted Averaging Operators: Theory and Applications, R.R. Yager and J. Kacprzyk (eds). Kluwer Academic Publisher, 73-85

[8] Grabisch M. (1997) k-order additive discrete fuzzy measures and their representation. Fuzzy Sets and Systems 92, 167-189

[9] Grabisch M., Marichal J.-L., Roubens M. (2000) Equivalent representations of set functions. Mathematics of Operations Research, to appear

[10] Grabisch M., Roubens M. (1999) An axiomatic approach to the concept of interaction among players in cooperative games. Int. J. of Game Theory, to appear 
[11] Marichal J.-L. (1998) Aggregation operators for multicriteria decision aid. Ph.D. Thesis, University of Liège, Liège, Belgium

[12] Marichal J.-L. (1999) Aggregation of interacting criteria by means of the discrete Choquet integral. Preprint 9910, GEMME, Faculty of Economics, University of Liège, Liège, Belgium

[13] Marichal J.-L. (1999) The influence of variables on pseudo-Boolean functions with applications to game theory and multicriteria decision making. Preprint 9916, GEMME, Faculty of Economics, University of Liège, Liège, Belgium

[14] Marichal J.-L., Roubens M. (1999) Determination of weights of interacting criteria from a reference set. European Journal of Operational Research, to appear

[15] Murofushi T. (1992) A technique for reading fuzzy measures (I): the Shapley value with respect to a fuzzy measure. In: 2nd Fuzzy Workshop, Nagaoka, Japan, October 1992, 39-48. In Japanese

[16] Murofushi T., Soneda S. (1993) Techniques for reading fuzzy measures (III): interaction index. In: 9th Fuzzy System Symposium, Sapporo, Japan, May 1993, 693-696. In Japanese

[17] Murofushi T., Sugeno M. (1989) An interpretation of fuzzy measure and the Choquet integral as an integral with respect to a fuzzy measure. Fuzzy Sets and Systems 29, $201-227$

[18] Murofushi T., Sugeno M. (1991) A theory of fuzzy measures. Representation, the Choquet integral and null sets. Journal of Mathematical Analysis and Applications $159 / 2,532-549$

[19] Rota G.C. (1964) On the foundations of combinatorial theory I. Theory of Möbius functions. Zeitschrift für Wahrscheinlichkeitstheorie und Verwandte Gebiete 2, 340-368

[20] Shannon C.E. (1948) A mathematical theory of communication. Bell System Tech. J. 27, 379-423, 623-656

[21] Shannon C.E., Weaver W. (1949) A mathematical theory of communication. University of Illinois Press, Urbana

[22] Shapley L.S. (1953) A value for $n$-person games. In: H.W. Kuhn and A.W. Tucker (eds). Contributions to the Theory of Games, Vol. II, Annals of Mathematics Studies, 28. Princeton University Press, Princeton, NJ, 307-317

[23] Sugeno M. (1974) Theory of fuzzy integrals and its applications. Ph.D. Thesis, Tokyo Institute of Technology, Tokyo

[24] Yager R.R. (1983) Entropy and specificity in a mathematical theory of evidence. Int. J. Gen. Systems 9, 249-260

[25] Yager R.R. (1988) On ordered weighted averaging aggregation operators in multicriteria decision making. IEEE Trans. on Systems, Man and Cybernetics 18, 183-190 
[26] Yager R.R. (1996) Quantifier guided aggregation using OWA operators. Int. J. Intelligent Systems 11, 49-73 